\title{
LA TRADUCCIÓN PARA EL DOBLAJE DEL VERNACULAR AFROAMERICANO: EL CASO DE THE CORNER (I): MARCO TEÓRICO Y CONTEXTO \\ Borja Aranda Molina \\ Universidad de Málaga
}

\begin{abstract}
Translation for dubbing of African American Vernacular: the case of The Corner (I): theoretical framework and context

The aim of this article is to analyze translation of the linguistic variation in the TV show The Corner. Throughout this study, we will take into account what must be considered for the translation of the linguistic variation; the speech variety which is used by the speakers in the show - the African American Vernacular - and translation for dubbing. Finally, we analyze some cases from the dubbed version and how the series translator has faced them and offer some translation suggestions for particularly relevant cases. Our conclusion is that the main difficulty of dubbing texts with a strong linguistic variation is their cultural idiosyncrasy and ethnical identity, hard to translate into languages from non-segregated cultures.
\end{abstract}

KEYWORDS: African American Vernacular, linguistic variation, The Corner, standard Spanish

\section{RESUMEN}

El siguiente artículo estudiará la traducción de la variación lingüística en la miniserie The Corner. Estudiaremos qué se tiene que tener en cuenta para la traducción de la variación lingüística; la variedad lingüística presente en la serie, el vernacular afroamericano, y la traducción para el doblaje. Por último, se estudiarán diferentes casos aparecidos en la serie, cómo el traductor se ha enfrentado a ellos y propuestas de traducción de casos concretos, concluyendo que la principal dificultad del doblaje de textos con fuerte carga de variación lingüística es la idiosincrasia cultural e identidad étnica propias de estos, que resultan de difícil trasvase a la lengua de culturas no segregadas ni identificadas con los mismos problemas raciales.

PALABRAS CLAVE: Vernacular afroamericano, variación lingüística, The Corner, español estándar

Fecha de envío: 06/04/2018

Fecha de revisión: 23/05/2018

Fecha de aceptación: 24/07/2018

Páginas: $129-137$ 


\section{INTRODUCCIÓN}

El vernacular afroamericano es una variedad lingüística de difícil estudio; su constante evolución a lo largo del siglo Xx e incluso en el presente hace que sea necesario una constante revisión de las posiciones desde las que se estudia. Su carácter evolutivo, por ende, provoca que la tarea de traducir esta variedad sea más compleja, ya que lo que a lo mejor hace 15 años parecía una propuesta firme, hoy en día, debido a la también constante evolución y adaptación del lenguaje vulgar, quede anticuada.

El nombre de la serie cuyo caso se estudia en este artículo, The Corner (traducible a «La esquina»), hace mención al lugar donde tienen lugar todos los trapicheos en los barrios de Baltimore, un microcosmos en el que la variación lingüística del vernacular afroamericano aparece en su máxima expresión y los usuarios se expresan con su propio sociolecto, su jerga sobre la droga y su utilización de palabras tabú propias. La serie apareció a principios de este siglo, momento en el que el doblaje de la variación lingüística era uno de los mayores retos para la traducción, y más en televisión, donde no se trabajaba tan concienzudamente en la calidad de los doblajes debido a su menor relevancia en aquel momento. Esto ofrece la posibilidad de estudiar cómo se podría haber mejorado aquella primera traducción.

Estudiaremos si hay alguna posibilidad más adecuada que la del español estándar que se repite en la serie y en el caso contrario, cómo se podría trabajar el léxico de una manera más natural y actual. Pero para poder estudiar la metodología utilizada por el traductor, antes tendremos que comprender conceptos sobre la variación lingüística, su traducción y los principales problemas que conlleva; también será necesario comprender las circunstancias que rodean al vernacular afroamericano: su nacimiento, evolución, estado actual y las principales diferencias y similitudes con las otras variedades principales del inglés, así como sus rasgos morfológicos, sintácticos, fonológicos y léxicos principales; uno de los últimos pasos antes de pasar a estudiar la traducción de The Corner será comprender las condiciones especiales que supone la traducción para el doblaje, y los elementos que el traductor debe tener en cuenta en esta especialidad de traducción para no acabar obteniendo una traducción conflictiva o incorrecta desde un plano político y social.

Por último, mediante una tabla comparativa del texto original y su doblaje, se compararán determinados ejemplos para sacar en claro aquellos más conflictivos y, a ser posible, que sean ejemplos de un buen trasvase lingüístico o todo lo contrario. Con esto, se busca encontrar una metodología que ayude a enfrentar esta variedad de la manera más adecuada y a la vez coherente con la idiosincrasia que caracteriza a la cultura objetivo.

Así, se pretende obtener una forma de actuar ante esta variedad que sea políticamente correcta y aceptable; que no resulte estereotípica ni degradante. Es decir, conocer en última instancia qué alternativas se pueden ofrecer en la traducción de esta variedad lingüística y cuáles son las mejores para evitar caer en un racismo estructural.

\section{BREVE INTRODUCCIÓN DE CONCEPTOS GENERALES DE LA VARIACIÓN LINGÜÍSTICA}

Como explica Roberto Mayoral (1999), el objeto de estudio de la variación lingüística y en concreto la sociolingüística es la relación entre la variación y el cambio lingüístico. Para establecer esta relación intervienen criterios de análisis y caracterización extralingüísticos (ideología, opresión, etc.) que analizan la variación como manifestación de desigualdades sociales y de relaciones de poder con los que se pueden proponer modificaciones del habla como forma de incidir en un cambio social favorable a los discriminados socialmente. Por lo tanto, el estudio de la variación lingüística puede servir como herramienta para abrir paso a variedades maltratadas, como es el caso del vernacular afroamericano.

En esta línea, Mayoral propone el concepto de variación cultural: la transmisión de un mensaje en la traducción con situaciones y estrategias diferentes, cuando la situación del enunciado original no 
resulta igual de familiar para la lengua de destino. Es decir, el traductor deberá intentar que la diferencia cultural no se haga más explícita de lo necesaria o de lo intencionado en el texto original.

Para esto, es necesario analizar la variación lingüística, y así obtener una metodología que ofrezca pasos para enfrentarse a textos marcados por una variación lingüística. La traducción va más allá de la búsqueda de palabras correspondientes a cada lengua; más bien, las palabras solo son elementos secundarios en el discurso global y es el tono del texto el que tiene mayor importancia, ya que a veces contiene mucho más significado que las mismas palabras. Como defiende Seguinot (1997: 109), la traducción final dependerá de los factores externos que definan a cada traductor y de las estrategias que decida tomar para realizar dicha traducción, sin ninguna solución absoluta, dado el gran número de posibilidades disponibles.

Por esto, es crucial conocer la variedad lingüística que se estudia, su base histórica y lingüística: en nuestro caso, el vernacular afroamericano. Afroamericano.

\section{EL VERNACULAR AFROAMERICANO}

El vernacular afroamericano (African-American Vernacular English) antes conocido como vernacular negro americano (Black English Vernacular) es la variedad sociolectal y étnica de los hablantes afroamericanos de los Estados Unidos. Es la variedad sociolingüística del inglés utilizada prácticamente por todos los personajes de The Corner, llena de expresiones muy informales que pueden dificultar la comprensión de sus palabras si no se conoce de antemano.

Para Juan Villena (2016), las reglas que funcionan en esta variedad y su contraste con las reglas de las demás variedades (inglés estándar, o inglés no estándar blanco norteamericano) inducen a pensar en diferencias esenciales en su estructura subyacente. Sin embargo, Mufwene (1998) afirma que son los ideólogos los que sostienen que los afroamericanos hablan vernacular afroamericano, lo que provocó que blancos norteamericanos vieran el vernacular afroamericano como algo peculiar; esto provocó que un gran número de lingüistas se posicionasen a favor de representarlo como un dialecto normal del inglés, gobernado por normas como cualquier otra variedad. Pero para entender esto mejor, es importante comprender su historia.

\subsection{NACIMIENTO E HISTORIA DEL VERNACULAR AFROAMERICANO}

Según Wolfram y Torbert (2006), hay dos explicaciones principales que dominan el debate sobre el origen y desarrollo del vernacular afroamericano. Primero, la «hipótesis anglicista» argumenta que el origen del vernacular afroamericano puede rastrearse a las mismas fuentes que los primeros dialectos europeos del inglés que aparecieron en Estados Unidos: las variedades del inglés habladas en las islas británicas. Esta posición asume por lo tanto que los esclavos simplemente aprendieron las variedades regionales y sociales de los grupos de hablantes blancos más cercanos a medida que aprendían inglés y que durante las siguientes generaciones solo unos pocos rastros de las lenguas predecesoras permanecieron.

A mediados de los años 60 y los años 70, la posición anglicista fue cuestionada por la «hipótesis criolla». Los investigadores de lenguas criollas observaron que la situación lingüística de los esclavos africanos era diferente a la de los europeos. De hecho, las circunstancias extremas de subordinación y segregación llevaron al desarrollo de la lengua criolla, una lengua adaptada especialmente y formada por grupos que no compartían una lengua común para comunicarse. Este criollo se extendió por las plantaciones del sur de Estados Unidos, y se convirtió en un prototipo para el desarrollo del vernacular afroamericano. El punto de vista «criollista» sostiene que la lengua de los afroamericanos en Norteamérica ha cambiado mucho durante los siglos, pero que la huella de su pasado criollo todavía puede encontrarse en muchas características de esta variedad, de las que hablaremos más tarde.

Por otra parte, Baugh (2006) sostiene que el legado lingüístico de la trata de esclavos africanos se ha malentendido en Estados Unidos y en todo el mundo. Muchos de los estereotipos lingüísticos que se 
La traducción para el doblaje del vernacular afroamericano: el caso de the corner (I): marco teórico ...

les atribuyen a los afroamericanos son engañosos y exagerados; los descendientes de esclavos americanos no constituyen en ningún momento un grupo lingüístico homogéneo. Por esto, los afroamericanos que nacieron en zonas rurales hablan diferente a aquellos que nacieron en barrios pobres de ciudades. Esta posición se acerca a la de Mufwene (1998), que afirma que cada variedad evoluciona localmente, sujeta a presiones ecológicas propias. Sin embargo, los descendientes de esclavos comparten una historia lingüística única que los separa de aquellos cuyos antepasados no fueron africanos esclavizados. La explicación para esta circunstancia lingüística es que solo se importaron a Estados Unidos esclavos africanos y cuando era posible los traficantes de esclavos separaban a aquellos que hablaran la misma lengua. Esta práctica pretendía parar cualquier tipo de comunicación entre los esclavos para prevenir levantamientos durante los viajes por el Atlántico.

Aunque haya miles de afroamericanos que dominan el inglés estándar, es fácil olvidar la deslocalización producida por la esclavitud que hizo mucho más difícil para los descendientes de esclavos adquirir los mismos conocimientos. Mientras que la mayoría de inmigrantes tuvieron el lujo de poder compartir una lengua minoritaria que no fuera inglés a su llegada, este no era el caso para los esclavos.

Se podría concluir que el vernacular afroamericano ha recibido influencia tanto del contexto local en Estados Unidos como de su herencia lingüística, por lo que es difícil elegir una de las dos posiciones (anglicista o criollista) como acertada. Wolfram y Torbert (2006) optan por cederles a ambos bandos parte de la razón, y presentar una posición que admita ambas llamada «hipótesis de substrato» («Substrate Hypothesis»).

\subsection{ESTADO ACTUAL DEL VERNACULAR AFROAMERICANO}

El vernacular afroamericano es una variedad lingüística en constante evolución, de ahí que su desarrollo moderno sea casi más estimulante que su historia. Los estudios actuales ${ }^{1}$ muestran que los rasgos característicos del vernacular afroamericano son ahora más predominantes y que se ha convertido en una variedad transregional cuya distinción étnica es más importante que hace un siglo.

Los primeros rastros de su desarrollo actual aparecen en el sudeste de Estados Unidos a finales del siglo XIX, donde Jim Crow institucionalizó la segregación racial y adoptó leyes discriminatorias que prohibían a los afroamericanos, que apenas unos años antes habían sido liberados de su esclavitud, usar las mismas instalaciones que los blancos y conseguir los mismos trabajos o los mismos salarios. La principal consecuencia de esto fue la Gran Migración Negra de 1910 a 1970, que llevó a más de 6 millones de afroamericanos a huir de los estados sureños a ciudades del norte y del oeste en busca de mejores condiciones de vida. Aunque los afroamericanos no fueron linchados en sus destinos, acabaron viviendo en guetos apartados de la población blanca, en los que prácticamente siguieron hablando igual que en el sudeste de Estados Unidos. El simple hecho de que en el norte y oeste de Estados Unidos consideraran el sudeste subdesarrollado no ayudó, especialmente cuando se pensaba, como cuenta Mufwene (2014), que los blancos del sur hablaban de aquella manera porque sus antepasados habían recibido influencia de sus niñeras negras.

Durante la mitad del siglo pasado se desarrolló un sentido de identidad étnica vinculado al vernacular afroamericano, que supuso una fuerte oposición a las «normas blancas». Esto es una indicación del duro enfrentamiento cultural entre las instituciones blancas dominantes y la gente de color en la sociedad estadounidense, lo que ha contribuido a que sea siempre una variedad de actualidad. Como Wolfram y Torbert (2006: 231) afirman a este respecto:

There is no greater testament to the durability of African American culture than the vitality of the past and present voice of African American English.

${ }^{1}$ Wolfram, W. y Torbert, B. (2006). 


\subsection{RASGOS MORFOLÓGICOS Y SINTÁCTICOS DEL VERNACULAR AFROAMERICANO}

Los rasgos más característicos, propuestos por Wolfram y Schilling-Estes (1998: 229), son los siguientes:

Be como forma habitual, como en They be happy o She be staying at home. Muchos hablantes de esta variedad hacen una distinción entre estados temporales y habituales. Por lo tanto, They are happy y They be bappy no son sinónimos; el primero expresa una situación temporal mientras que el segundo expresa una situación habitual.

Ausencia de verbo copulativo para las contracciones de is y are (ejemplos: She nice. They acting all strange).

La pérdida de sufijos de inflexión como el $-s$ en verbos en presente y en tercera persona (ejemplos: she walk en vez de she walks, she raise en vez de she raises). En posesivos (man hat en vez de man's hat, Jack car en vez de Jack's car) y en plurales (a lot of time en vez de a lot of times).

El tiempo de pasado simple con had + verbo (They had went outside and then they had messed up the yard. Yesterday, she had fixed the bike and had rode it to school.

Ain't como forma de negación en vez de didn't. Esta no es exclusiva de los afroamericanos, y sí aparece más usada por el resto de la población por influencia lingüística.

Reducción de consonantes finales cuando van seguidas de una palabra que empieza por vocal (lif' up en vez de lift up, bus' up en vez de bust up) y cambios en consonantes iniciales. (skreet en vez de street, skraight en vez de straight).

Uso de [f] y [v] por la th final. (toof en vez de tooth, smoov en vez de smooth).

Uno de los rasgos más característicos tiene que ver con el contraste tonal. Muchas lenguas africanas expresan cambios de significado a través de cambios tonales y esto permite a sus hablantes expresar diferentes significados para la misma palabra dependiendo del tono o el énfasis. Por esto, no es de extrañar que se haya adoptado este contraste tonal. Baugh (2006: 222) ofrece el siguiente ejemplo para explicar este fenómeno:

If an African American student intended to say that He been sad was a temporary past event, they would intend for an unstressed form of been to be implied. However, if the intention was to convey that "He is not only sad at this moment, but he has been sad for quite some time," then a stressed form of been as in He BEEN sad would have been the intention.

También vemos un caso parecido con el uso de done para indicar una acción completa (He done went).

Siguiendo con el verbo to be, Green (1999) también observa que la tercera persona del singular de este auxiliar (is) se usa con todas las personas excepto la primera persona del singular: you, be, we, they IS running.

Por último, Martin y Wolfram (1998) destacan otra de las características más usuales en el vernacular afroamericano y otras variedades del inglés, que es la de la negación múltiple: el uso de dos o más morfemas de negación para comunicar una sola. Las negaciones múltiples se pueden dar en cualquier frase, tanto en la principal como en subordinadas, y no hay una posición particular que lleve la carga negativa, pero tiene que aparecer al menos en una posición desde la que se niegue la oración. Como explica Villena (2016), la doble negación es posible porque el inglés puede expresar la negación mediante dos procedimientos: la negación oracional, asociada a los sintagmas verbales, y la negación léxica, asociada a los sintagmas nominales (I ain't done nothing).

\subsection{RASGOS FONOLÓGICOS DEL VERNACULAR AFROAMERICANO}

Como las características fonológicas del vernacular afroamericano a veces se usan para llevar a niños al logopeda y otras veces son los ejemplos que los oyentes de esta variedad utilizan para 
La traducción para el doblaje del vernacular afroamericano: el caso de the corner (I): marco teórico ...

discriminarla, la fonología es un punto clave que abordar para entender algunos de los problemas sociales más intransigentes que sufren los afroamericanos. Como el sistema vocal proporciona una base más sólida para un análisis diacrónico que la mayoría de los rasgos morfosintácticos, la fonología es integral para entender los orígenes y el actual desarrollo del vernacular afroamericano. Por eso es importante conocer la tabla fonológica que presentan Bailey y Thomas (1998: 88):

\begin{tabular}{|c|c|}
\hline Característica & Ejemplos \\
\hline Pérdida de la consonante final & Cold $\rightarrow$ col_ [coul]; hand $\rightarrow$ han_ [hæn] \\
\hline Pérdida de sílaba átona & $\begin{array}{l}\text { About } \rightarrow \text { 'bout [bout]; government } \rightarrow \\
\text { gov'ment }\end{array}$ \\
\hline Haplología & Mississippi $\rightarrow$ [mIsIpI] \\
\hline Pérdida de /r/ después de consonante & 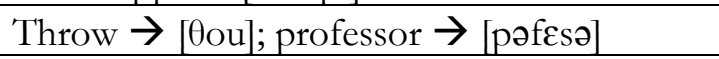 \\
\hline Labialización de fricativa interdental & Bath $\rightarrow$ [bæf] \\
\hline Fricativa inicial interrumpida & Those $\rightarrow$ [douz] \\
\hline Interrupción de interdental fricativa muda & Tenth $\rightarrow$ [tent]; with [wIt] \\
\hline Metátesis de /s/ final & Ask $\rightarrow$ [æks]; grasp $\rightarrow$ [græps] \\
\hline Vocalización o perdida de /r/ intervocálica & 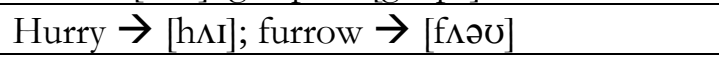 \\
\hline Vocalización de /r/ silábica & Bird $\rightarrow$ [b3d]; burr $\rightarrow[\mathrm{b} 3]$ \\
\hline Vocalización de /r/ después de vocal & Four $\rightarrow$ [fəə]; Ford $\rightarrow$ [foəd] \\
\hline Unión de vocales posteriores antes de /1/ & Bale $\rightarrow$ [beəl]; feel $\rightarrow$ [fiəl] \\
\hline Interrupción de fricativa antes de nasal & Isn't $\rightarrow$ [Idn]; wasn't [wpdn] \\
\hline Acentuación de silabas iniciales & Pólice $\rightarrow$ [pou'lis]; Detroit [ditroIt] \\
\hline Reducción de nasal final a vocal nasalizada & Man $\rightarrow[\mathrm{m} x]$ \\
\hline Pérdida de consonante final & Five $\rightarrow$ fi_[fa:]; fine $\rightarrow$ fi_[fa:] \\
\hline Desonorización de interrupción final & $\mathrm{Bad} \rightarrow[\mathrm{bæt}]$ \\
\hline Pérdida de /j/ después de consonante & $\begin{array}{l}\text { Computer } \rightarrow \text { [kəmpurə]; Houston } \rightarrow \\
\text { [hustn] }\end{array}$ \\
\hline Sustitución de /k/ por /t/ en /str/ & Street $\rightarrow$ [skrit]; stream $\rightarrow$ [skrim] \\
\hline
\end{tabular}

Tabla I. Rasgos fonológicos del vernacular afroamericano

De todas estas características, las más significativas y que parecen ser únicas del vernacular afroamericano son las 5 últimas.

\subsection{PECULIARIDADES LÉXICAS DEL VERNACULAR AFROAMERICANO}

Lo que dota de una naturaleza única al vernacular afroamericano son tres áreas principales que Smitherman (1998) recoge: primero, los patrones gramaticales y de pronunciación vistos anteriormente; segundo, ciertas ritualizaciones verbales que provienen de la tradición oral y cultural en culturas africanas; y tercero, el léxico, que se desarrolla a medida que se le da especial significado a ciertas palabras inglesas, una práctica común desde la esclavitud debido a la necesidad de crear un sistema de comunicación que solo estos esclavos pudieran entender.

Una de las expresiones más representativas del léxico del vernacular afroamericano es la de nigga. Spears (1998) aclara que el uso de nigga (a partir de ahora N) no tiene por qué tener necesariamente connotaciones racistas, y que términos como este se usan muy frecuentemente para referirse a individuos sin ninguna implicación secundaria. A veces incluso se usa con implicaciones positivas.

Muchos estadounidenses saben que $\mathrm{N}$ se usa mucho entre afroamericanos, pero estos lo toman como una grave ofensa si los blancos lo utilizan. Su uso más común es entre afroamericanos jóvenes para referirse simplemente a «hombre». Por lo tanto, el significado de $\mathrm{N}$ no cambia de ninguna manera cuando se aplica a personas de diferente etnia. De hecho, la gran mayoría de afroamericanos usan $\mathrm{N}$ cuando están con otros afroamericanos, y lo usan a pesar de sus posturas en cuanto al uso de N; algunos pueden estar en contra de su uso, pero lo usan de todas maneras. 
Podemos distinguir usos neutrales en los que $\mathrm{N}$ simplemente sirve de sinónimo de «guy, dude, homeboy, homie, brotha' o partna', que podrían traducirse al español como «tío, colega, hermano, compañero», etc. En The Corner veremos constantemente el uso de $\mathrm{N}$, que podría traducirse por cualquiera de las anteriormente propuestas. El uso de $\mathrm{N}$ lleva consigo una vulgarización del léxico y una bajada de registro que justifica que The Corner esté cargada de palabras malsonantes.

\section{LA TRADUCCIÓN PARA EL DOBLAJE EN ESPAÑOL DE THE CORNER}

Los personajes de The Corner, naturales de Baltimore, en Maryland, están basados en personas reales de las que se documentó David Simon para el libro de corte periodístico del mismo título que escribió junto a Ed Burns. La serie, al pretender ser lo más realista posible, procura que sus personajes actúen y hablen igual que sus homólogos reales. Además, al principio de cada capítulo un entrevistador hace preguntas a los personajes ficticios, lo que marca más si cabe la pretensión realista que enmarca la miniserie. Estos personajes son hablantes del vernacular afroamericano.

A lo largo de la miniserie, y como se ejemplificará posteriormente, el traductor opta por la estandarización de la variedad lingüística; es decir, los personajes hablan en una variedad estándar del español, pero con un registro muy informal y jerga urbana. Al encontrarnos con esto, la pregunta principal que cabe hacerse es la siguiente:

\section{1 ¿POR QUÉ OPTA EL TRADUCTOR POR LA ESTANDARIZACIÓN DEL VERNACULAR AFROAMERICANO?}

Para poder entender la motivación que lleva al traductor a utilizar una variedad estandarizada hay que volver de nuevo a Mayoral (1999) y la definición que ofrece de las pistas de contextualización: elementos del enunciado que permiten a cada receptor asignar los parámetros sociolingüísticos del contexto; es decir, no expresan exclusivamente la variación, sino que incluyen también la ubicación espaciotemporal de la producción y publicación del texto que se estudie, la identidad de los participantes en el acto de comunicación para el que ha sido producido, etc. En primer lugar, el traductor tiene que interpretar la información sobre la obra y el autor y, en segundo lugar, la información previa relacionada con el contenido, como la época, el tema, los personajes u otros textos asociados, para así poder realizar una traducción coherente y adecuada.

Un afroamericano utilizará pistas de contextualización muy codificadas para caracterizar a otro afroamericano, y esas pistas estarán basadas en el conocimiento objetivo de los estereotipos. Sin embargo, un blanco español caracterizando a un afroamericano utilizará pistas poco codificadas dado que el estereotipo del afroamericano en España está basado en muy pocos elementos marcadores y responderán en gran medida a realidades no observadas directamente. Un caso característico puede ser el del hablante español caracterizando a un negro norteamericano mediante rasgos que identifica como propios del español hablado por negros caribeños.

Esto, que no son más que clichés, a veces tiene cierta base objetiva (la sustitución del sonido de /r/ por el de /l/ para hablantes chinos), pero en otros casos no corresponde con la realidad que pretenden mostrar (la caracterización de los indios americanos conjugando todos los verbos en infinitivo o el uso de la variedad andaluza para la traducción del vernacular afroamericano).

Las pistas de contextualización deben estar basadas en marcadores reconocibles por los destinatarios y, como Mayoral resume, sustituir los elementos del estereotipo conocido por elementos extraídos de la realidad objetiva cuando esta no resulta familiar puede llevar a que la comunicación sea un fracaso, y esta es una de las razones principales por las que el traductor de The Corner pueda optar por una estandarización. Se puede establecer en general que los diferentes tipos de pistas presentan diferentes grados de dificultad al traductor, siendo más fácil trabajar con las pistas léxicas y más difícil trabajar con las fonéticas, por lo que resulta lógico que el traductor opte por una vulgarización del léxico y un registro más bajo, en pos de mantener una variedad fonética estándar de acuerdo con la capacidad lingüística 
potencial del grupo de destinatarios, ya que estas representaciones estereotípicas finalmente forman parte del acervo lingüístico de los hablantes.

Como explica Marset (2009: 3): «el doblaje es un fenómeno que no se limita a la traducción, sino que incluye otro tipo de operaciones que estrictamente hablando no son traductológicas». La lengua a la que se traduce no tiene por qué subordinarse a la lengua origen, sino que tiene que reproducir el mismo mensaje mediante los medios expresivos que le son propios. Por eso es crucial tener en cuenta cual será el público de esa traducción.

La mejor manera de escapar de la estandarización quizá sea adaptar el léxico de la manera que resulte más natural. Por esto, Chaume (2012) concluye que, dado que las variedades no tienen equivalentes en otras lenguas, y que coincidir una variedad con otra de la lengua de destino puede resultar políticamente incorrecto, el traductor debe optar por no sustituir una variedad por otra. Este autor recomienda usar un registro no estándar para así mostrar que el/los personaje/s hablan una variedad no estándar. Usar una variedad que podría ser asociada a clase baja o a cualquier otro grupo desfavorecido tiene que evitarse en la medida de lo posible; aunque no sea una solución perfecta, no compromete al traductor, a pesar de que se pierda el efecto particular que la variedad consigue en el producto original. El reto al que se enfrenta el traductor es entender las connotaciones ideológicas y políticas que ofrece la variedad para realizar la traducción más fiel posible.

The Corner está plagada de palabras tabú y expresiones coloquiales, dobles negaciones, frases sin sujeto, interrogativas sin el auxiliar do e interrogativas sin inversión de la frase, omisiones, pérdida de nasalización y otras características lingüísticas del vernacular afroamericano. En este caso, resulta necesario emular en la medida de lo posible el dialecto de los afroamericanos en la lengua de destino. La equivalencia se podrá obtener con elecciones léxicas de bajo registro donde aparezca una desviación fonética, o con palabras no comunes para reflejar una desviación léxica; esto es, jugando con la fonética, la gramática y el léxico cuando sea necesario y compensándolo en toda la serie.

Con toda esta información, se trabajó en una tabla comparativa de la versión original y su doblaje a lo largo de los seis episodios que componen The Corner para estudiar y analizar los casos más representativos, así como proponer nuevas opciones que cumplan lo anteriormente descrito. Este análisis compone el grueso principal de la siguiente parte del artículo.

\section{BIBLIOGRAFÍA}

Baugh, John (2006): «When Linguistic Worlds Collide (African American English)» en Wolfram, Walt y Ben Ward: American Voices: How dialects differ from coast to coast. Oxford, Blackwell Publishing.

Cambrigde University Press (2008): Cambridge online dictionary [en línea]: http://dictionary.cambridge.org/es/.

Chaume, Frederic (2012): Audiovisual translation: Dubbing. Manchester, St. Jerome Publishing.

Marset, Mabel Richart (2009): La alegría de transformar. Valencia, Tirant Lo Blanch.

Martin, Sarah. et al. (1998): African-American English: Structure, history and use. New York, Routledge.

Mayoral, Roberto (1999): Traducción de la variación lingüistica. Soria, Diputación provincial de Soria.

Merriam-Webster Dictionary [en línea]: https://www.merriam-webster.com/.

Mufwene, Salikoko (2014): «The English origins of African American Vernacular English: What Edgar W. Schneider has taught us» en Buschfeld, Sarah. et al.: The Evolution of Englishes. The Dynamic Model and beyond. Amsterdam, John Benjamins Publishing Company, [en línea]: http://mufwene.uchicago.edu/publications/ENGLISH\%20ORIGINS\%20OF\%20AAE\%20_ \%20FEST\%20FOR\%20EDGAR\%20SCHNEIDER\%202014.pdf. [Consulta: 2 de febrero de 2018].

Real Academia Española (2001): Diccionario de la lengua española. (22. ${ }^{a}$ ed.) [en línea]. http://dle.rae.es/?w=diccionario.

Seguinot, Candance (1996): Accounting for variability in translation. London, Sage Publications.

Urban Dictionary [en línea]: http://www.urbandictionary.com. 
Villena Ponsoda, Juan Andrés (2016): «Variación lingüística y traducción: por qué el traductor necesita del variacionista» en Caprara, Giovanni, Emilio Ortega y Juan Andrés Villena: Variación lingüistica, traducción y cultura: De la conceptualización a la práctica profesional. New York, Peter Lang.

Wolfram, Walt y Natalie Schilling-Estes (1998): «American English: Dialects and Variation» en Trudgill, Peter: Language in Society. Friburgo, Blackwell Publishing.

Wolfram, Walt y Benjamin Torbert, (2006): «Bridging the Great Divide (African American English)» en Wolfram, Walt y Ben Ward: American Voices: How dialects differ from coast to coast. Oxford, Blackwell Publishing.

Wolfram, Walt (2004): The grammar of urban African American Vernacular [en línea]: http://citeseerx.ist.psu.edu/viewdoc/summary?doi=10.1.1.516.25. [Consulta: 2 de febrero de 2018]. 